\title{
DISCUSSION SUR LA POSITION TAXONOMIQUE D'APHALLOIDES COELOMICOLA DOLLFUS, GHABAUD ET GOLVAN, 1957 (TREMATODA, OPISTHORCHIOIDEA)
}

\author{
CH. BAYSSADE-DUFOUR* et C. MAILLARD**
}

RÉSUMÉ. Aphalloides coelomicola a été attribué tantôt aux Cryptogonimidae, tantôt aux Heterophyidae, tantôt aux Opisthorchiidae.

Sa chétotaxie cercarienne est nettement différente de celle des Heterophyidae et de celle des Opisthorchiidae ; elle est si proche de celle des Acanthostomidae que nous concluons à l'appartenance d'Aphalloides à cette famille, bien que nous ne connaissions pas encore la chétotaxie des Cryptogonimidae.

\section{Discussion on the taxonomic position of Aphalloides coelomicola Dollfus, Chabaud and Golvan, 1957 (Trematoda, Opisthorchioidea).}

SUMMARY. Aphalloides coelomicola has been assigned to the Cryptogonimidae, Heterophyidae or the Opisthorchiidae by previous authors. Cercarial chaetotaxy is very different from that of Heterophyidae and Opisthorchiidae but very similar to that of Acanthostomidae. Althouth the chaetotaxy of Cryptogonimidae is unknown, we propose that the genus Aphalloides belongs to the Acanthostomidae.

Dollfus, Chabaud et Golvan (1957) décrivent un Trématode : Aphalloides coelomicola qu'ils qualifient d' " aberrant au point de vue systématique " mais dont l'anatomie permet le rattachement aux Opisthorchioidea.

Cette super-famille comprend 4 familles : Acanthostomidae Poche, $1926^{1}$. Heterophyidae Odhner, 1914. Opisthorchiidae Braun, 1901. Cryptogonimidae, Ciurea, 1933.

La position préovarienne du réceptable séminal d'Aphalloides l'apparente aux Acanthostomidae et aux Heterophyidae et l'éloigne des Opisthorchiidae ; son absence

* Muséum National d'Histoire Naturelle, Laboratoire de Zoologie des Vers, associé au CNRS, 61 rue Buffon, F 75231 Paris Cedex 05.

** Université des Sciences et Techniques du Languedoc, Laboratoire de Parasitologie Comparée, place Eugène Bataillon, F 34060 Montpellier Cedex.

I. Le taxon supragénérique le plus ancien étant celui des Anisocoeliinae Looss, rgor, cette famille devrait s'appeler Anisocoeliidae pour être conforme aux lois de la nomenclature. Nous conservons cependant le nom d'Acanthostomidae que l'usage semble avoir consacré.

Accepté le I $_{4}$ mai 1982. 
de gonotyl le rapproche des Opisthorchiidae et le différencie des AcanthostomidaeHeterophyidae.

Dollfus et coll. le placent donc provisoirement dans la famille hétérogène des Cryptogonimidae chez laquelle le réceptable séminal est préovarien ou postovarien et le gonotyl présent ou absent. Sur la base d'autres critères, Naidenova (1970) attribue ce Ver aux Heterophyidae et Yamaguti (1971) aux Opisthorchiidae. Brooks (1980) donne une révision des Acanthostominae qu'il considère comme une sousfamille des Cryptogonimidae.

Maillard (1973) réalise le cycle biologique d'Aphalloides coelomicola et démontre qu'il s'agit d'un parasite à deux hôtes. La cercaire est de type parapleurolophocerque comme cela s'observe chez les Opisthorchioidea ; la métacercaire progénétique, localisée dans le coelome, a une morphologie aberrante.

Bayssade-Dufour (1979) décrit la chétotaxie cercarienne correspondante (fig. 31, $32,34,35 \mathrm{~A}, 42 \mathrm{~A}$ et $42 \mathrm{G})$.

Pour tenter de classer ce genre énigmatique nous avons à comparer cette chétotaxie à celle de neuf autres cercaires d'Opithorchioidea actuellement décrites qui appartiennent à trois familles :

- famille des Heterophiidae avec Apophallus donicus (Skrjabin et Lindtrop, 1919) et A. muehlingi (Jägerskiold, 1889) (cf. Odening, 1973), Euryhelmis squamula (Rud, 1819) (cf. Combes, Jourdane et Richard, 1974), Haplorchis pumilio (Looss, 1896) (cf. Bayssade-Dufour et Ow-Yang, 1975) ;

- famille des Opisthorchiidae avec Clonorchis sinensis (Cobbold, 1875) (cf. BayssadeDufour, Albaret et Dong Cham Kim, 1982) ;

- famille des Acanthostomidae avec Timoniella imbutiforme (Acanthostomum imbutiforme (Molin, 1859); et T. praeterita (Looss, 1901) (cf. respectivement Maillard, 1973 et Bayssade-Dufour, 1979).

La chétotaxie caudale de la cercaire d'Aphalloides coelomicola est identique à celle de :

- deux Acanthostomidae : Timoniella imbutiforme et T. praeterita,

— un Heterophyidae : Euryhelmis squamula,

- un Opisthorchidae : Clonorchis sinensis.

ce qui est une preuve supplémentaire de l'appartenance d'Aphalloides à la superfamille des Opisthorchioidea.

Par contre la chétotaxie céphalique et corporelle de la cercaire est totalement différente de ce que l'on observe chez les Heterophyidae et l'Opisthorchiidae : $C$. sinensis, mais ressemble beaucoup à celle des deux Timoniella: $T$. imbutiforme et $T$. praeterita (Acanthostomidae) ( fig 1).

- Aphalloides et les deux Timoniella ont en commun : $1 \mathrm{C}_{\mathrm{II}} 1,2 \mathrm{C}_{\mathrm{III}} 1,2 \mathrm{C}_{\mathrm{III}} 2,1 \mathrm{C}_{\mathrm{IV}} 1$, $2 \mathrm{~A}_{\mathrm{I}} \mathrm{V}, 1 \mathrm{P}_{\mathrm{I}} \mathrm{D}, 1 \mathrm{P}$ IIIV, 1 PIII, 6 UL et 2 UD.

- Aphalloides a en commun avec $T$. imbutiforme: $3 \mathrm{C}_{\mathrm{II}} 3,2 \mathrm{~A} \mathrm{II}, 1 \mathrm{MD}$.

- Aphalloides a en commun avec $T$. praeterita: $1 \mathrm{C}_{\mathrm{IV}} 2,1 \mathrm{P}_{\mathrm{II}} \mathrm{D}$.

- Aphalloides diffère des deux Timoniella aux niveaux $\mathrm{C}_{\mathrm{I}}, \mathrm{A}_{\mathrm{I}} \mathrm{D}$ et $\mathrm{A}_{\mathrm{L}}-\mathrm{P}_{\mathrm{L}}$. 

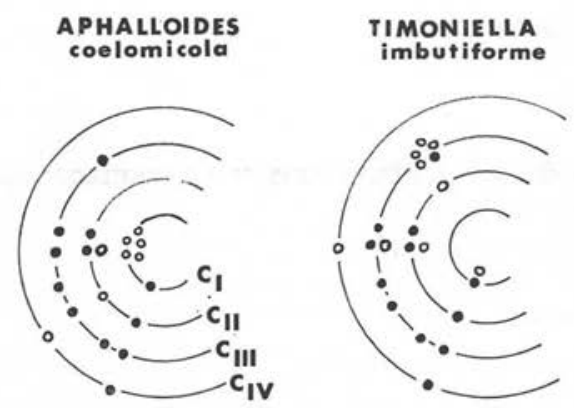

\section{TIMONIELLA praeterita}
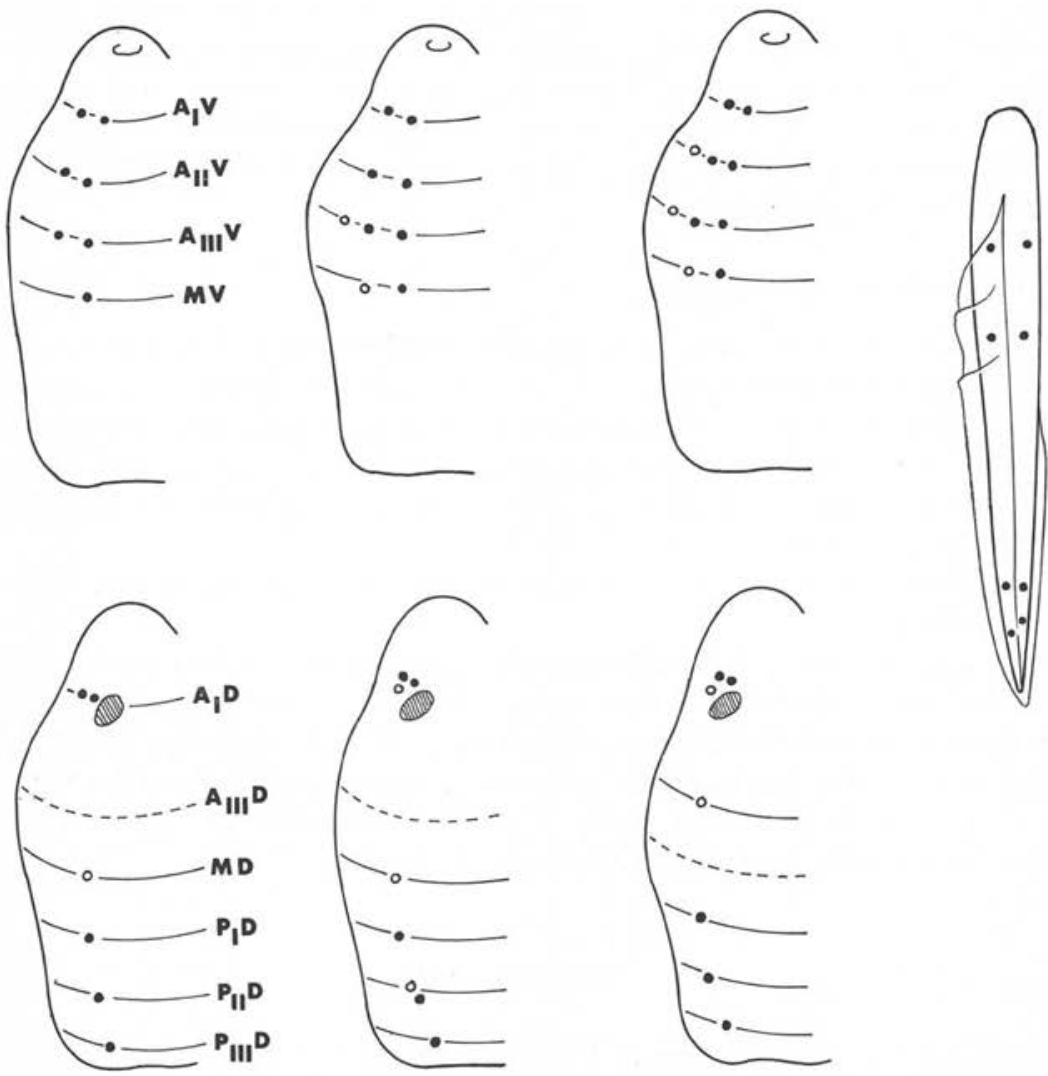

FIG. I. - Chétotaxie comparée d'Aphalloides coelomicola, Timoniella imbutiforme et Timoniella praeterita. Les papilles en noir sont communes aux trois espèces, la chétotaxie caudale est identique chez les trois espèces. 


\section{Discussion}

\section{Morphologie de l'adulte.}

Dollfus et coll. (1957) admettent que deux caractères sont très importants au point de vue systématique chez les Opisthorchioidea :

- La position préovarienne ou postovarienne du réceptacle séminal,

- la présence ou l'absence de gonotyl (Aphalloides a un réceptacle séminal préovarien et n'a pas de gonotyl).

Dollfus et coll. admettent que la position préovarienne du réceptacle séminal s'accompagne toujours de la présence d'un gonotyl chez les Acanthostomidae et en est indépendante chez les Cryptogominidae.

En fait, lorsqu'on se réfère à la diagnose des Acanthostomidae donnée par Poche (1926) (in Yamaguti, 1971) et par Price (1940) on constate que chez cette famille le gonotyl peut être présent ou absent : "gonotyls may be present " et "gonotyls absent in adults ». La conclusion selon laquelle Aphalloides coelomicola appartiendrait aux Acanthostomidae (Poche, 1926) est donc compatible avec la diagnose fondée sur les caractéristiques de l'adulte.

\section{Chétotaxie de la cercaire.}

La chétotaxie céphalique et corporelle d'Aphalloides coelomicola est très différente de celle des Heterophyidae et de celle des Opisthorchiidae; l'appartenance du genre Aphalloides à l'une ou l'autre de ces deux familles n'est donc pas possible.

La chétotaxie céphalique et corporelle d'A Ahalloides est, par contre, si proche de celle des deux Acanthostomidae que nous connaissons qu'il est possible d'attribuer ce genre à cette famille.

Au sujet de l'appartenance éventuelle d'Aphalloides aux Cryptogonimidae, notre position est la suivante :

- ou bien la chétotaxie des Cryptogonimidae, lorsqu'elle sera connue, sera différente de celle des Acanthostomidae et dans ce cas Aphalloides n'est pas un Cryptogonimidae, - ou bien la chétotaxie des Cryptogonimidae sera suffisamment proche de celle d'Aphalloides et des autres Acanthostomidae, que la famille des Cryptogonimidae (sous-famille Cryptogoniminae Ward, 1917) sera synonyme de celle des Acanthostomidae (sous-famille Acanthostominae Nicoll, 1914).

\section{BIBLIOGRAPHIE}

BAysSAde-Dufour Ch. : L'appareil sensoriel des cercaires et la systématique des Trématodes digénétiques. Mém. Mus. Nat. Hist. nat. A. Zool., I979, II3, I-8I.

Bayssade-Dufour Ch., Albaret J. L., Dong Cham Kim : Chétotaxie cercarienne d'une souche coréenne des Clonorchis sinensis. Présence vraisemblable de ce Trématode en Afrique. Ann. Parasitol. Hum. Comp., I982 (à paraître).

Bayssade-Dufour Ch., Albaret J. L., Ow-Yang C. K. : Sensilles et protonéphridies de Centrocestus formosanus et Centrocestus sp. (Trematoda, Heterophyidae, Centrocestinae). Ann. Parasitol. Hum. Comp., r982 (à paraître). 
BAysSADE-Dufour Ch., Ow-YANG C. K. : The sensory receptors of two cercaria form Malaysia : Trichobilharzia brevis (Schistosomatidae) and Haplorchis pumilio (Heterophyidae). The Southeast Asian J. Trop. Med. Pub. health, 1975, 6, 338-342.

Brooks D. R. : Revision of the Acanthostominae Poche 1926 (Digenea : Cryptogonimidae). Zool. J. Linnean Soc., 1980, 70, 313-382.

Combes C., Jourdane J., Richard J. : Recherches sur le cycle biologique de Euryhelmis squamula (Rudolphi, I8I9) parasite de Neomys fodiens dans les Pyrénées. Z. Parasitkde, 1974, $44,81-92$.

Dollfus R. Ph., Chabaud A. G., Golvan Y. J. : Helminthes de la région de Banyuls. V. Nouveau Distome Aphalloides coelomicola n. gen. n. sp. de la cavité générale d'un Gobius d'eau saumâtre. Ann. Parasitol. Hum. Comp., 1957, 32, 28-40.

Maillard C. : Mise en évidence du cycle évolutif abrégé d'Aphalloides coelomicola Dollfus, Chabaud et Golvan, 1957 (Trematoda). Notion " d'hôte historique ». C.R. Acad. Sci. Paris, $1973,227 D: 317-320$.

Naidenova N. N. : Parasitofaune des Gobiidae de la Mer Noire et de la Mer d'Azov. Biologie de la mer (20 Naukova dumka, Kiev), 1970, 74-113 (en russe).

OdenING K.: Der Lebenszyklus des Trematoden Apophallus donicus in Berlin im Vergleich zu A. muehlingi. Biol. Z Zbl., 1973, 92, 455-494.

Poche F. : Das system der Platodaria. Arch. Naturgesch, I926. Abt. A (2) : I-240; (B) : 24I-458.

PRICE E. W. : A review of Trematode superfamily Opisthorchioidea. Proceed. Helminth. Soc. Washington, $1940,7,1-13$.

Yamaguti S. : Synopsis of Digenetic Trematodes of Verbebrates, Keigaku Pub. Co, Tokyo, 1971, I-1074. 